SLAC-PUB-8034-Rev

March, 1998

\title{
A Generalised Area Law for Hadronic String Reinteractions*
}

\author{
Johan Rathsman \\ Stanford Linear Accelerator Center \\ Stanford University, Stanford, California 94309, USA \\ e-mail: rathsman@slac.stanford.edu
}

\begin{abstract}
A new model for hadronic string reinteractions based on a generalised area law is presented. The model describes both the hadronic final states in $e^{+} e^{-}$ annihilation and the diffractive structure function in deep inelastic scattering. The model also predicts a shift in the W-mass reconstructed from hadronic decays of $\mathrm{W}$-pairs of the order $65 \mathrm{MeV}$.
\end{abstract}

*Work supported by the Swedish Natural Science Research Council, contract F-PD 11264-301 and the U.S. Department of Energy, contract DE-AC03-76SF00515. 
The problem addressed in this letter is how to translate a partonic final state consisting of quark and gluons, calculated in perturbative quantum chromo dynamics (QCD), into a final state of hadrons. Since this a process that takes place at low momentum transfer, perturbative methods cannot readily be applied. Instead one has to take resort to phenomenological models, like the Lund string model [1], to describe this non-perturbative transition. The string model is a semi-classical model which, when combined with perturbatively calculated partonic cross-sections, gives a good overall description of observed hadronic final states, especially in $e^{+} e^{-}$collisions.

One of the basic assumptions of the Lund string model is that the colour field between two colour charges forms a flux-tube with the dynamical properties of a one-dimensional relativistic string. In the simplest case with just one quark and one anti-quark connected by a string this gives the so called yo-yo model. Another assumption which is usually made in the application of the Lund string model is that two different strings will hadronise independently of each other even if they overlap. In other words there is no cross-talk or string reinteractions in a system consisting of more than one string.

This letter presents a new general model for taking string reinteractions into account in hadronic final states. String reinteractions as a way of understanding diffractive events was suggested a long time ago [2]. Similar ideas have also been considered in connection with W-pair production [3-11] and in the model for soft colour interactions (SCI) which originally was formulated for rapidity gaps in deep inelastic scattering [12.13].

The starting point for the present model will be the string configuration given by the colour flow in a reaction on partonic level where the parton configuration has been calculated perturbatively. On top of that interactions in the form of string rearrangements will be added. Thus, giving an altered colour configuration and thereby a different hadronic final state. The main difference compared to SCI is that the model presented here considers string reinteractions where the colour fields in the two strings interact whereas in SCI the perturbative partons interact with a hadronic background field.

The model. The Lund String model is based on the so called area law [14]. Simply put the area law means that configurations with a large area are exponentially suppressed. Within the Lund String model the probability for a configuration with area $A$ is given by $P \propto \exp (-b A)$ where $b$ is a phenomenological parameter of the order $0.6 \mathrm{GeV}^{-2}$ if the area is calculated in energy-momentum coordinates. In the following, the area for a piece of string spanned between two partons $p_{i}$ and $p_{j}$ will be calculated as $A_{i j}=\left(p_{i}+p_{j}\right)^{2}-\left(m_{i}+m_{j}\right)^{2}=$ $2\left(p_{i} p_{j}-m_{i} m_{j}\right)$ such that the area vanishes for two massive partons at rest. This way of defining the area reduces to the ordinary $A=\left(E_{i}+p_{z, i}\right)\left(E_{j}-p_{z, j}\right)=\left(E+\left|p_{z}\right|\right)^{2}$, in the center of mass system with the partons along the $z$-axis, when the quark masses are neglected.

For a single string the only way to decrease the area is by "popping" quark pairs from the vacuum. However, for a system consisting of several strings it may also be possible to decrease the area by doing a string rearrangement. The simplest example is given by the situation where there are two strings, each consisting of a quark anti-quark pair as illustrated in Fig. 国. Labeling the momenta of the partons in the two initial strings $\left(p_{1}, p_{2}\right)$ and $\left(p_{3}\right.$, $p_{4}$ ) respectively it may be possible that the system can decrease its area by making a string rearrangement into $\left(p_{1}, p_{4}\right)$ and $\left(p_{2}, p_{3}\right)$. The initial area is given by $A^{\text {old }}=A_{12}+A_{34}$ where as the alternative configuration has the area $A^{\text {new }}=A_{14}+A_{23}$.

Generalising the area law to be applicable to a system of strings the probabilities for 

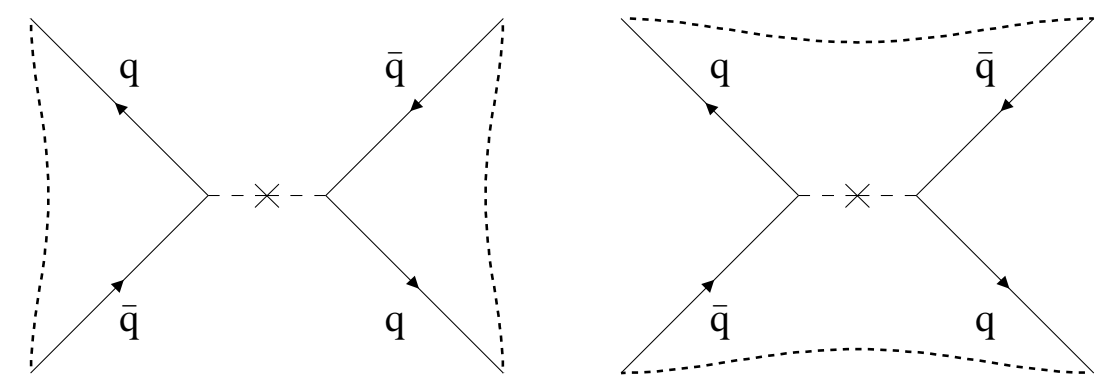

FIG. 1. Illustration of two different string configurations in $\mathrm{W}$-pair production in the lowest order (the strings are indicated with dashed lines).

these two different configurations are given by $\exp \left(-b A^{\text {old }}\right)$ and $\exp \left(-b A^{\text {new }}\right)$ respectively times some overall factor depending on the parton momenta which is the same for both of them. In this paper it will be assumed that the probability for a string rearrangement is proportional to the normalised difference between the two configurations

$$
P \propto \frac{\exp \left(-b A^{\text {new }}\right)-\exp \left(-b A^{\text {old }}\right)}{\exp \left(-b A^{\text {new }}\right)}=1-\exp (-b \Delta A)
$$

where $\Delta A=A^{\text {old }}-A^{\text {new }}$ is the area difference between the old and the new configuration.

In addition there should also be a colour suppression factor of the order $\frac{1}{N_{C}^{2}}$ reflecting the required colour matching of the two strings. Since in this model it is the strings that are thought to interact it is the colour field in the string that is relevant and not the colour charges at the endpoints of the string. This should be compared with the fact that in a quark-gluon-anti-quark string the quark and the anti-quark are not in a colour singlet state. The string carries an octet charge and thus the colour factor required for matching the colour field in the two strings is of the order $\frac{1}{N_{C}^{2}}$.

The phenomenological ansatz of the model is to have a string rearrangement between a pair of string pieces with the probability

$$
P=R_{0}[1-\exp (-b \Delta A)]
$$

where $R_{0}$ is a nonperturbative parameter of the order $\frac{1}{N_{C}^{2}}$. The parameter will be fixed to $R_{0}=0.1$ by comparing with data on rapidity gap events in deep inelastic scattering. This formula for the probability should be compared with the SCI model where the probability is assumed to be a constant, i.e. without the suppression factor $[1-\exp (-b \Delta A)]$. The

\footnotetext{
${ }^{1}$ In an earlier version of the model the string-length was used to formulate a suppression factor. I would like to thank Gunnar Ingelman for suggesting to use the string-area instead when deriving the suppression factor.
} 
actual implementation of the string rearrangements in the model is very similar to the one used for the SCI model. In short there is a loop over all pairs of string pieces which have an alternative string configuration. A string rearrangement between the two string pieces is then made with the probability given by Eq. (2). In principle one has to worry about the fact that the order of the pairs in the loop can make a difference. However, since the probability to make a string rearrangement is small, this is a minor problem and the effects have been neglected. The model is implemented in the Lund Monte Carlo (MC) framework and the code can be obtained from http://www3.tsl.uu.se/thep/rathsman/gal/.

Electron-positron annihilation. Since in this model the string rearrangement is thought of as a general phenomenon and not connected with some hadronic background field as in SCI [12,13] it has first of all to be retuned to data from $e^{+} e^{-}$annihilation at $\sqrt{s}=M_{Z}$. For this purpose the Jetset Monte Carlo version 7.4 [15] will be used together with the model. For easy reference most of the times the default version of Jetset will be used for comparison instead of data and differences smaller than a few percent will be considered satisfactory.

The model is tuned using the particle multiplicities and momentum distributions. By retuning the parameter $b$ in the fragmentation function to $b=0.45 \mathrm{GeV}^{-2}$ and the cut-off in the parton showers to $Q_{0}=2 \mathrm{GeV}$, the mean multiplicity of charged particles $\left(<n_{c h}>=20.9\right)$ as well as the dispersion $\left(D=\sqrt{\left.\left\langle n_{c h}^{2}\right\rangle-<n_{c h}\right\rangle^{2}}=6.2\right)$ are the same as in the default version. The resulting charged multiplicity distribution is shown in Fig. 2(a) together with the default version of Jetset and data from the ALEPH collaboration [16]. The multiplicities for individual mesons and baryons are typically within a percent of the default version.

Considering the momentum distributions the differences are typically of the order a few percent which is about the size of the errors in the data. As an example Fig. Z(b) shows the $\pi^{ \pm}$momentum distribution for the new model with and without retuning compared to default Jetset and also compared with data from the OPAL collaboration [17]. As can be seen from the figure the new model is actually closer to the data than the default version and it should be possible to get a good agreement with data after a dedicated retuning.

With the charged multiplicity and the momentum distributions used to retune the model, the string effect and the rapidity gap distribution will be used to check if the model is a viable alternative to the normal string model as implemented in Jetset. The string effect [18 20] gives a measure of the colour structure in three-jet events in $e^{+} e^{-}$annihilation. In the the lowest order diagram $e^{+} e^{-} \rightarrow q \bar{q} g$ the string goes from the quark via the gluon to the anti-quark. In turn this means that there will be a depletion of particles produced between the two quark jets compared to the particle production between the gluon jet and either of the quark jets since there is no string between the quarks.

To study the string effect the JADE algorithm was used for jet reconstruction with the resolution $y_{\text {cut }}=0.05$. The events giving three jets were analysed by projecting all particles on to the plane spanned by the two most energetic jets. Fig. 3(a) shows the particle flow in this plane as a function of the angle $(\omega)$ from the most energetic jet with $\omega$ defined such that the second jet has $\omega<\pi$. With the least energetic jet normally being the gluon jet this shows the relative depletion between the two quark jets. As can be seen from the figure the difference between the default version of Jetset and the model is very small (below a few percent as shown by the lower histogram giving the ratio of the two). For illustration the 

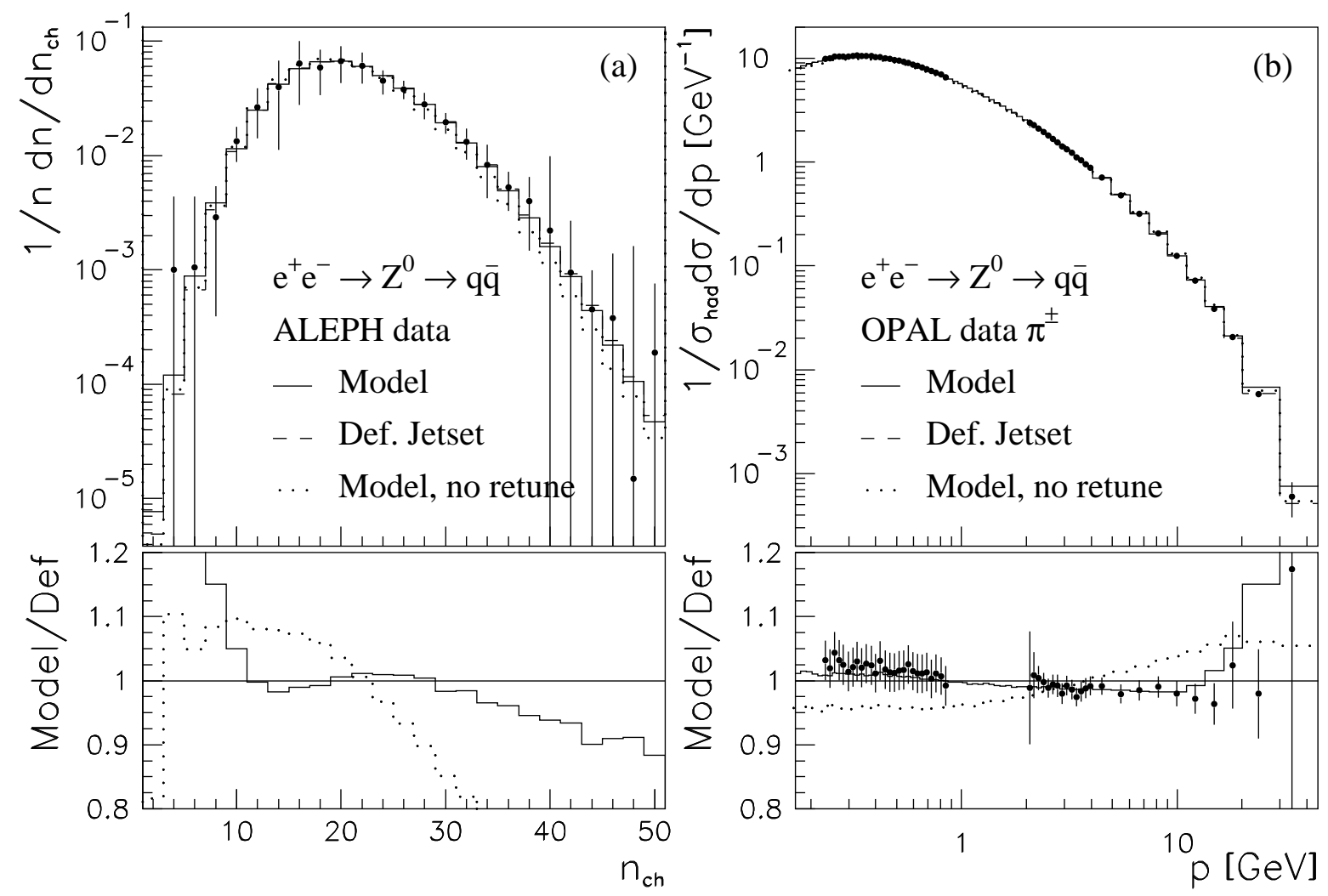

FIG. 2. The charged multiplicity distribution (a) and the momentum distribution for $\pi^{ \pm}$(b). The model (solid line) is compared with default Jetset (dashed line) and the model without retuning (dotted line). The data are from the ALEPH and OPAL collaborations respectively with the statistical and systematic errors added in quadrature. The ratio of the model, with (solid) and without (dotted) retuning, to default Jetset is shown below, (b) also shows the ratio of data to default Jetset. 

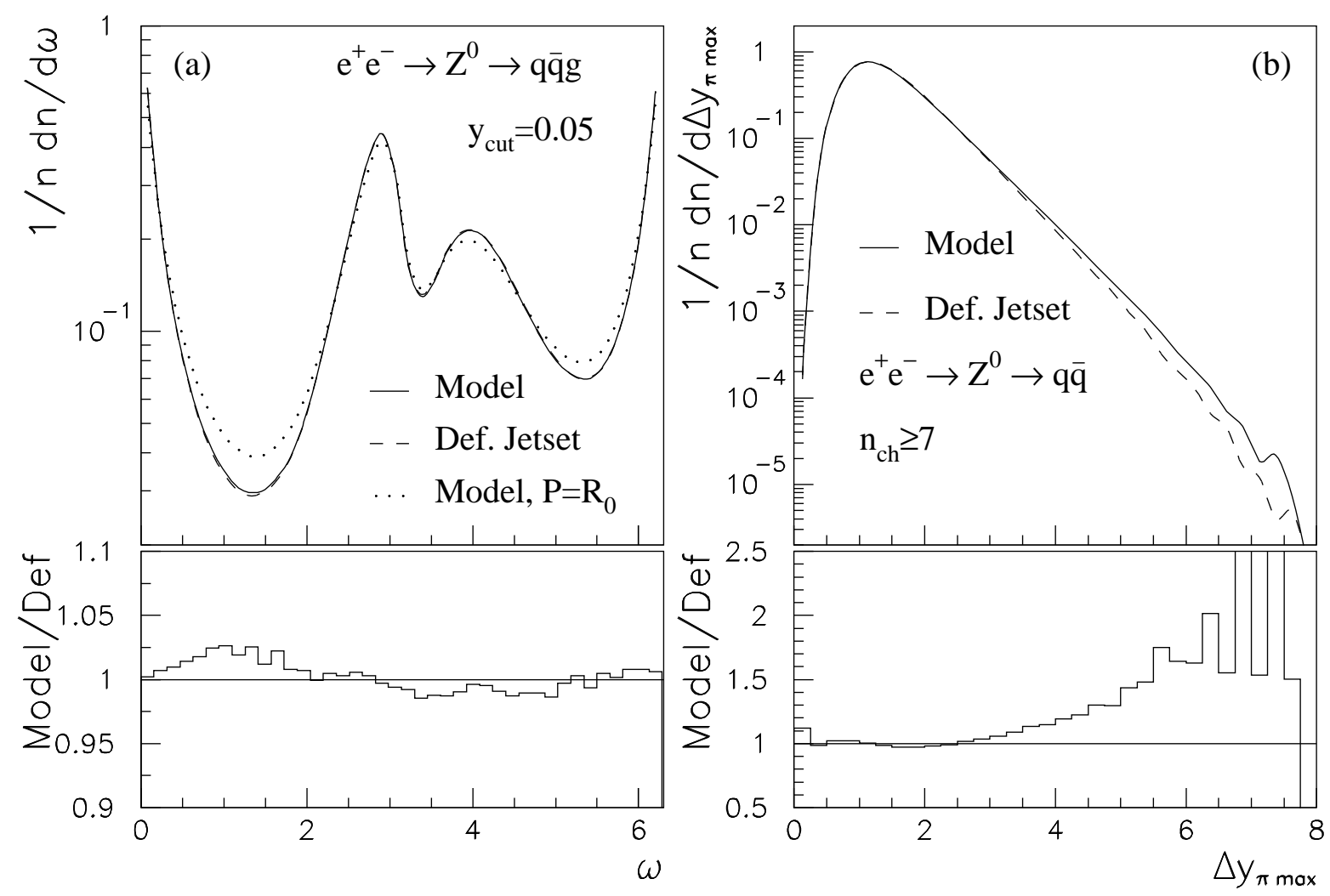

FIG. 3. (a) Illustration of the string effect as explained in the text and (b) the largest rapidity gap distribution. The model (solid line) is compared with default Jetset (dashed line) and in (a) the model without the area suppression factor (dotted line). The ratio of the model and default Jetset is shown below.

model without the area suppression factor is also shown.

Another observable which is sensitive to the colour structure of an event is the rapidity gap distribution. In $e^{+} e^{-}$annihilation the rapidity is usually defined as $y=\frac{1}{2} \ln \frac{E+p_{z}}{E-p_{z}}$, where the $p_{z}$ is the momentum along the thrust axis and $E$ is the energy. The rapidity gap distribution has been measured by the SLD collaboration [21] where they assumed the mass

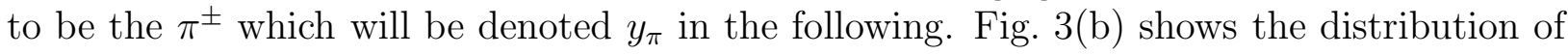
the largest gap in an event $\Delta y_{\pi \max }$. As can be seen from the figure, the difference between the model and the default version of Jetset is small for $\Delta y_{\pi \max }<4$ but for larger values the difference grows and becomes as large as a factor 2 for $\Delta y_{\pi \max }>6$. However, judging by figure 1 in [21] the errors in the data points in this region are of the same size or even larger so at this point it is not possible to draw any conclusions from the existing measurement? The important point to notice is that the model does not give any plateau in the rapidity gap

\footnotetext{
${ }^{2}$ The SLD collaboration has approximately three times more data on tape which is in the process of being analysed [22].
} 
distribution as one might expect but only a gradual decrease of the slope in the exponential suppression of large gaps.

Diffractive deep inelastic scattering. As already mentioned the free parameter in the model $R_{0}=0.1$ is determined by calculating the diffractive structure function in deep inelastic scattering and comparing with data from the $\mathrm{H} 1$ collaboration [23]. The calculation is done by implementing the model in the Lepto Monte Carlo [24] version 6.5 with the CTEQ 4 leading order parton distributions [25]. The only changes with respect to the default version of Lepto is to use the same values for the cut-offs in the initial and final state parton showers $\left(Q_{0}=2 \mathrm{GeV}\right)$ and the hadronisation parameter $\left(b=0.45 \mathrm{GeV}^{-2}\right)$ as for $e^{+} e^{-}$annihilation given above?. The diffractive structure function was then evaluated using a subroutine from the HzTool package [26].

The agreement between the resulting diffractive structure function calculated from the model and the $\mathrm{H} 1$ data is very good as is shown in Fig. 4, especially if one takes into account that there is only one free parameter in the model. Both the so called Pomeron exchanges which are thought to dominate for small $x_{\mathbb{I P}}$, with $x_{\mathbb{P P}}$ being the longitudinal momentum of the Pomeron with respect to the protonf, as well as the other Regge exchanges which are important in the transition region $0.01<x_{\mathrm{IP}}<0.1$ are explained by the model. The model only fails for small masses of the diffractive system $M_{X}^{2}=Q^{2} \frac{1-\beta}{\beta}$ which are not included in the model because of the cut-off $M_{X}^{2}>4 \mathrm{GeV}^{2}$ in the matrix-element.

$\mathrm{W}$-pair production. The precise measurement of the $\mathrm{W}$-mass is an important test of electroweak theory. At LEP2 W-pairs are produced close to threshold in $e^{+} e^{-}$annihilation. One way of measuring the $\mathrm{W}$-mass directly is to reconstruct it from hadronic decays. When both of the W's decay hadronically one has to take into account the possibility that the two hadronic systems interfere with each otherf. This was first studied in [3] whereas the effects on the W-mass was first considered in [4]. Later there have been several different models suggested for modeling the effects of colour reconnections on the reconstructed W-mass [5-11]. For a recent comparison of different models with data see e.g. [27,28].

In the present model, which has been implemented using the Pythia Monte Carlo [15] version 5.7, similar effects are expected. Fig. 5(a) shows the dijet mass spectrum for W-pairs (produced in $e^{+} e^{-}$annihilation at $\sqrt{s}=183 \mathrm{GeV}$ ) decaying hadronically using the JADE algorithm for jet reconstruction with $y_{c u t}=0.015$ and considering only those events giving four jets. Fitting a Breit-Wigner form plus a constant gives a reconstructed W-mass which is $65 \pm 15 \mathrm{MeV}$ larger in the model than in the default Pythia version (the error is statistical). This gives an estimate of how large a mass shift one can expect even though the precise

\footnotetext{
${ }^{3}$ In addition version 2 of the sea-quark treatment (see [13]) was used with the width of the mean virtuality set to $0.44 \mathrm{GeV}$. However, the result is not sensitive to this choice.

${ }^{4}$ With the mass of the diffractive system denoted by $M_{X}$, the photon virtuality $Q^{2}$ and $\mathrm{W}$ being the mass of the hadronic system, $x_{\mathrm{IP}} \simeq \frac{Q^{2}+M_{X}^{2}}{Q^{2}+W^{2}}$ and $\beta \simeq \frac{Q^{2}}{Q^{2}+M_{X}^{2}}$.

${ }^{5}$ The separation, at LEP2, between the two W's before they decay is small $(\sim 0.1 \mathrm{fm})$ on a hadronic scale.
} 


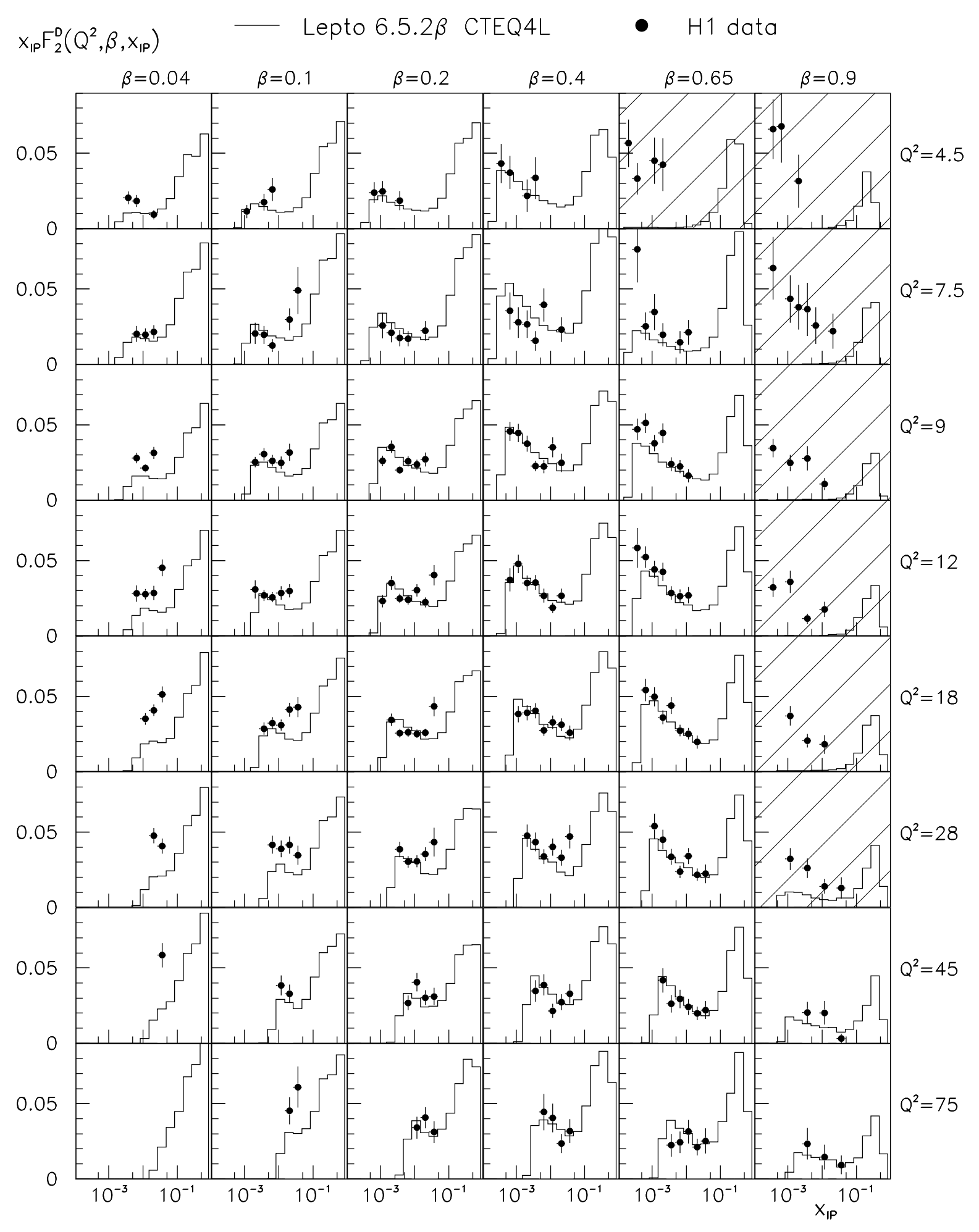

FIG. 4. The diffractive structure function obtained with the model applied to Lepto compared to data from the $\mathrm{H} 1$ collaboration. The hashed plots corresponds to kinematic points where the mass of the diffractive system $M_{X}$ is smaller than $2 \mathrm{GeV}$ which is the cut-off in the matrix element. 

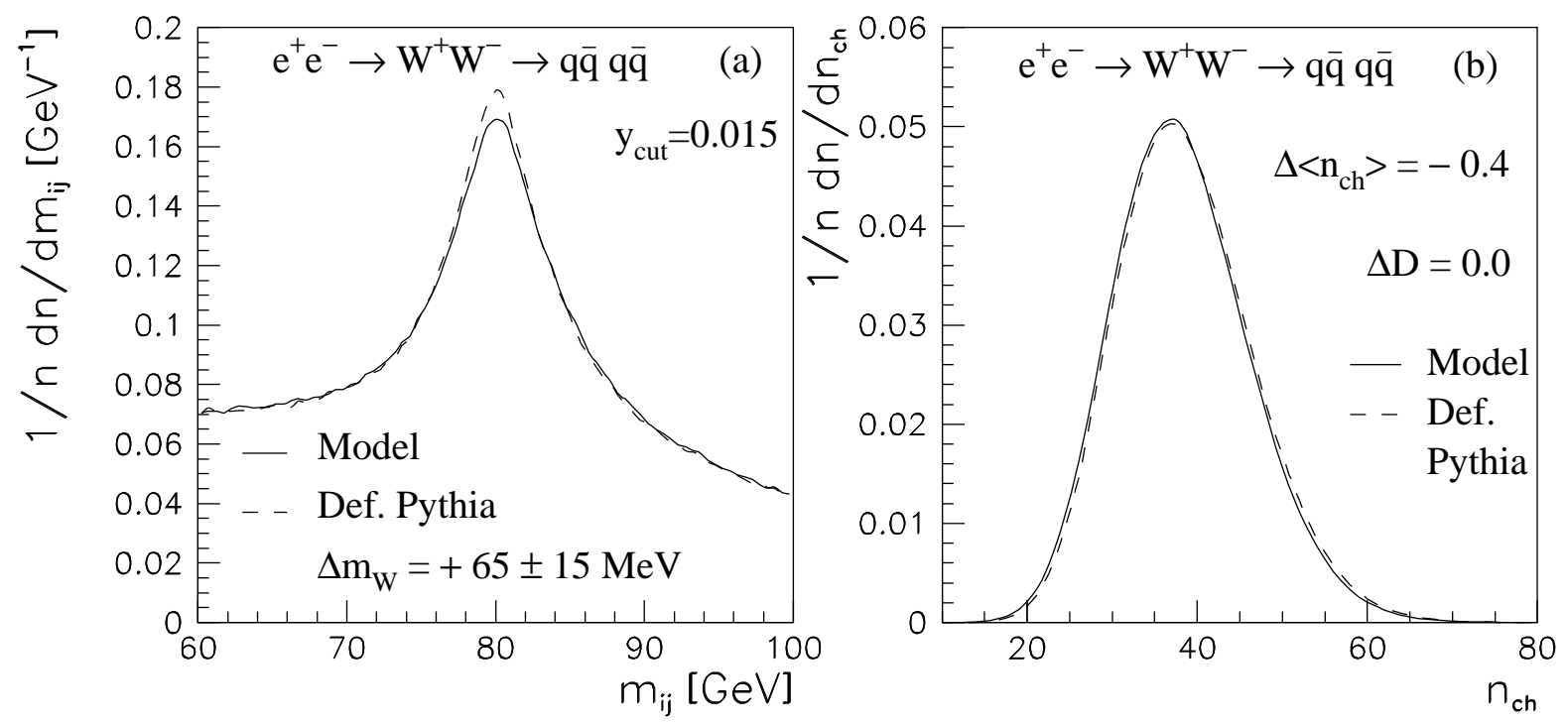

FIG. 5. The dijet mass spectrum (a) and the charged multiplicity (b) for W-pairs decaying hadronically produced in $e^{+} e^{-}$annihilation at $\sqrt{s}=183 \mathrm{GeV}$. The model (solid) is compared with default Pythia (dashed). As indicated, the reconstructed mass is shifted with $\Delta m_{W}=m_{W}^{\text {Model }}-m_{W}^{\text {Def }}=65 \pm 15 \mathrm{MeV}$, the mean multiplicity is shifted with $\Delta<n_{c h}>=<n_{c h}>^{\text {Model }}-<n_{c h}>^{\text {Def }}=-0.4$ whereas the dispersion $D=\sqrt{\left\langle n_{c h}^{2}>-<n_{c h}\right\rangle^{2}}$ is unchanged.

number will depend on the analysis method used.

The obtained mass-shift is approximately twice as big as the final statistical error for LEP2 predicted in [29]. On the one hand this could ruin the usefulness of the hadronic decays for an exact determination of the W-mass but on the other hand it could be used as a probe for non-perturbative dynamics. Other observables that have been used for comparing different models with data are the multiplicity and thrust distributions (see e.g. [27,28]). The multiplicity distribution in the present model is shown in Fig. 5(b). Compared with default Pythia, the mean multiplicity is somewhat smaller, $\left.\Delta<n_{c h}\right\rangle=-0.4$, whereas the dispersion is unchanged. The latter is also true for the thrust distribution.

Conclusions. A new general model for string rearrangements in hadronic final states has been presented. For hadronic final states in $e^{+} e^{-}$annihilation the model gives small differences compared to the Jetset Monte Carlo which in general describes data very well. Thus the model is a viable extension of the ordinary Lund string model. At the same time the model describes the diffractive structure function in deep inelastic scattering. The model also predicts an enhancement for large rapidity gaps in $e^{+} e^{-}$annihilation which in principle should be measurable with more statistics and a shift in the W-mass reconstructed from Wpairs decaying hadronically. With more data the model can be further tested and possibly provide a probe into nonperturbative QCD phenomena.

Acknowledgments. I would like to thank Gunnar Ingelman and Anders Edin for useful discussions on the modeling of non-perturbative effects in general, and Gunnar Ingelman 
for valuable comments on the manuscript. I would also like to thank Torbjörn Sjöstrand for helpful communications on the Lund string model and Hannes Jung for providing the HzTool routine for calculating the diffractive structure function. 


\section{REFERENCES}

[1] B. Andersson, G. Gustafson, G. Ingelman, T. Sjöstrand, Phys. Rep. 97 (1983) 31.

[2] X. Artru and G. Mennessier, Nucl. Phys. B70 (1974) 93.

[3] G. Gustafson, U. Pettersson and P.M. Zerwas, Phys. Lett. B209 (1988) 90.

[4] T. Sjöstrand and V. A. Khoze, Phys. Rev. Lett. 72 (1994) 28.

[5] T. Sjöstrand and V. A. Khoze, Z. Phys. C62 (1994) 281.

[6] G. Gustafson and J. Häkkinen, Z. Phys. C64 (1994) 659.

[7] L. Lönnblad, Z. Phys. C70 (1996) 107.

[8] J. Ellis and K. Geiger, Phys. Rev. D54 (1996) 1967.

[9] C. Friberg, G. Gustafson and J. Häkkinen, N. Phys. B490 (1997) 289.

[10] J. Ellis and K. Geiger, Phys. Lett. B404 (1997) 230.

[11] A. Donnachie and P. V. Landshoff, Phys. Lett. B421 (1998) 341.

[12] A. Edin, G. Ingelman, J. Rathsman, Phys. Lett. B366 (1996) 371.

[13] A. Edin, G. Ingelman, J. Rathsman, Z. Phys. C75 (1997) 57.

[14] K. G. Wilson, Phys. Rev. D10 (1974) 2445.

[15] T. Sjöstrand, Pythia 5.7 and Jetset 7.4, Comp. Phys. Comm. 82, 74 (1994).

[16] ALEPH Collaboration, D. Buskulic et al., Z. Phys. C69 (1995) 15.

[17] OPAL Collaboration, R. Akers et al., Z. Phys. C63 (1994) 81.

[18] B. Andersson, G. Gustafson and C. Peterson, Z. Phys. C 1 (1980) 105.

[19] B. Andersson and G. Gustafson, Z. Phys. C 3 (1980) 223.

[20] B. Andersson, G. Gustafson and C. Peterson, Phys. Lett. B101 (1981) 129.

[21] SLD Collaboration, K. Abe et al., Phys. Rev. Lett. 76 (1996) 4886.

[22] D. Muller, private communication.

[23] H1 Collaboration, C. Adloff et al., Z. Phys. C76 (1997) 613.

[24] G. Ingelman, A. Edin, J. Rathsman, LePto 6.5, Comp. Phys. Comm. 101, (1997) 108.

[25] CTEQ collaboration, MSUHEP-60416, CTEQ-604.

[26] N. Brook et al., 'HzTool- A Package for Monte Carlo Generator - Data Comparisons at HERA (version 1.0)', http://dice2.desy.de/ h01rtc/hztool.html.

[27] N. K. Watson, BHAM-HEP/98-01, hep-ph/9809564.

[28] OPAL Collaboration, G. Abbiendi et al., CERN-PPE-98-196, hep-ex/9901019.

[29] W mass working group, Z. Kunszt et al., in Physics at LEP2, eds. G. Altarelli, T. Sjöstrand and F. Zwirner,CERN report 96-01 (1996), Vol. 1, p.141, hep-ph/9602352. 